\title{
Estimación de la prevalencia, intensidad del dolor postoperatorio y satisfacción de los pacientes postoperados del Hospital Ángeles Lomas
}

\author{
Estimation of the prevalence, intensity of postoperative pain and \\ satisfaction of postoperative patients at Hospital Ángeles Lomas \\ Dulce Miriam Vargas Aguilar, * Elise Bañuelos Ortiz, ${ }^{*}$ Ludivina Asunción Cortés Martínez, * \\ Alondra Rocío Martínez Nápoles, ${ }^{\ddagger}$ Daria Irina Romero Luna, ${ }^{*}$ \\ Hitandehuitl Guevara Valerio, § Yvhone Luna Alonso I
}

\section{Resumen}

El control del dolor es un indicador de buena práctica y de calidad asistencial que influye en la satisfacción del paciente. La Sociedad Americana de Dolor recomienda sondear la satisfacción como resultado de la buena práctica clínica. Existe evidencia de que un adecuado alivio del dolor no sólo aumenta el bienestar y la satisfacción de los pacientes, sino que, además, contribuye a disminuir la morbilidad postoperatoria y reduce la estancia hospitalaria. Sin embargo, es poco lo que sabemos sobre su prevalencia en nuestra institución: el Hospital Ángeles Lomas. El objetivo de este estudio de tipo observacional, descriptivo y transversal fue conocer la prevalencia e intensidad del dolor postoperatorio de los pacientes operados. Material y métodos: Se solicitó la participación de 102 pacientes: 56 mujeres y 46 hombres a las 24 horas del postoperatorio para contestar el cuestionario de la Asociación Americana del Dolor de satisfacción con el manejo del dolor, siendo éste un instrumento validado y confiable. Para analizar las variables, se utilizó estadística descriptiva. Resultados: La prevalencia de dolor postoperatorio fue del $87.25 \%$ y la satisfacción global media de 9.24 en una escala de 0 a 10. Conclusión: Los resultados mostraron dolor leve y satisfacción global alta con el manejo del dolor postoperatorio.

Palabras clave: Dolor postoperatorio, satisfacción del paciente, prevalencia, intensidad del dolor, dolor en México.

* Anestesióloga del Hospital Ángeles Lomas. Ciudad de México.

* Residente del 1er. año de Algología del INCan. Ciudad de México.

$\S$ Anestesióloga del Hospital General de Chimalhuacán. Estado de México.

II Anestesióloga Algóloga del Hospital Ángeles Lomas y del Hospital IMSS Lomas Verdes. Ciudad de México.

Correspondencia:

Dra. Dulce Miriam Vargas Aguilar

Correo electrónico: dradulcevargas@outlook.com

Aceptado: 12-09-2019.

www.medigraphic.com/actamedica

\section{Abstract}

Pain control is an indicator of good practice and quality of care that influences patient satisfaction. The American Pain Society recommends surveying satisfaction as a result of good clinical practice. There is evidence that adequate pain relief not only increases the well-being and satisfaction of patients, but also contributes to reducing postoperative morbidity and reduces hospital stay, however, we know little about the prevalence in our institution: the Angeles Lomas Hospital. The aim of the observational, descriptive and cross-sectional study was to know the prevalence and intensity of postoperative pain in patients undergoing elective surgery. Material and methods: We requested the participation of 102 patients, 56 women and 46 men at 24 hours postoperatively, to answer the questionnaire of satisfaction with pain management of the American Pain Association, this being a validated and reliable instrument. Descriptive statistics was used. Results: The prevalence of postoperative pain was $87.25 \%$ and the mean global satisfaction was 9.24 in a 0 to 10 scale. Conclusion: The results showed mild pain and high overall satisfaction with postoperative pain management.

Keywords: Postoperative pain, patient satisfaction, postoperative pain prevalence, pain intensity, pain in Mexico.

\section{INTRODUCCIÓN}

El dolor es definido por la Asociación Internacional para el Estudio del Dolor como un una experiencia sensorial y emocional desagradable que se asocia con un daño tisular real o potencial, descrita en términos de tales daños. ${ }^{1,2} \mathrm{Al}$ ser el dolor una experiencia subjetiva, para su medición, se requiere de la información suministrada por el paciente, ya que los signos físicos y metabólicos son inespecíficos y no mantienen necesariamente una relación proporcional a la magnitud del dolor. ${ }^{2,3}$ Por esta razón, el manejo oportuno 
y efectivo del dolor agudo ha sido y es uno de los mayores desafíos de la medicina. ${ }^{3}$

La documentación de la intensidad del dolor debe realizarse con escalas que sean de fácil uso e interpretación. ${ }^{2,4}$ En la literatura médica, se han publicado escalas validadas como la escala visual analógica (EVA). ${ }^{2}$ Por un lado, para el dolor agudo perioperatorio, se considera que una puntuación de EVA de 1 a 4 indica dolor leve; de 5 a 7 , moderado, y de 8 a 10, severo. ${ }^{4}$ Por el otro, el dolor postoperatorio se define como aquél que aparece como una consecuencia del acto quirúrgico. ${ }^{2,5}$

Por su parte, la satisfacción del paciente está considerada como un elemento deseable e importante del actuar médico; ${ }^{3,4}$ esta evaluación de satisfacción será necesaria para mejorar los servicios prestados a los pacientes. ${ }^{6}$ Esta condición, aunque difícil de medir, informa sobre el proceso recibido durante su atención y puede ser la base para futuras recomendaciones. Por ello, en nuestro hospital se considera muy trascendente.

El control satisfactorio del dolor postoperatorio es uno de los retos más importantes que permanecen sin resolver en el ámbito quirúrgico, lo que conlleva a un fuerte impacto en los pacientes y en el sistema sanitario en su conjunto. ${ }^{7}$ Es una evidencia innegable que la mayoría de los pacientes que se someten a una intervención quirúrgica padecen dolor en un grado variable. ${ }^{8}$ En 1988, la Sociedad Americana de Dolor (SAD) estableció un comité para desarrollar guías clínicas para mejorar la calidad del manejo de dolor.9,10 La SAD recomendó que la satisfacción fuera encuestada como resultado de la práctica clínica y desarrollo de un modelo de encuesta de satisfacción. ${ }^{11}$ En el año 2015, en el Instituto Nacional de Ciencias Médicas y Nutrición en la Ciudad de México, se realizó un diagnóstico situacional con respecto al manejo del dolor postoperatorio ${ }^{12}$ mediante una adaptación del cuestionario internacional de dolor postoperatorio, denominado Pain Out, ${ }^{13}$ el mismo que se utilizó en este estudio (Anexo 1). Como se demostrado, un adecuado alivio del dolor no sólo aumenta el bienestar y la satisfacción de los pacientes, sino que además contribuye a disminuir la morbilidad postoperatoria y reduce la estancia hospitalaria. 2,11 Por esta razón, el objetivo de este estudio observacional fue conocer la prevalencia e intensidad del dolor postoperatorio y la satisfacción del manejo del dolor en pacientes postquirúrgicos de manera electiva en el Hospital Ángeles Lomas.

\section{MATERIAL Y MÉTODOS}

El estudio se llevó a cabo en el Hospital Ángeles Lomas. Éste se realizó con base en los términos de la declaración de Helsinki y Ginebra de la Asociación Médica Mundial, así como con la previa autorización del Comité Local de
Investigación y Ética del Hospital Ángeles Lomas. Una vez obtenido el consentimiento informado del paciente, se realizó el estudio.

Éste es un estudio observacional, descriptivo y transversal en el que se entrevistaron a 102 pacientes postquirúrgicos de tipo electivo en el área de quirófanos centrales durante el 1ero. de junio de 2017 al 30 de junio de 2017.

En este estudio, se incluyeron a pacientes mayores de 18 años de cualquier género, estado físico según la Sociedad Americana de Anestesiología (ASA) I-III, que hubieran estado en cirugía programada y que cumplieran las 24 horas postoperatorias. Se excluyeron a aquellos pacientes que se negaron a contestar el cuestionario, que fueran incapaces de comprender la escala para la evaluación del dolor, pacientes que recibieron el alta hospitalaria antes de 24 horas tras la intervención quirúrgica, pacientes que precisaron cuidados críticos en el postoperatorio inmediato y pacientes que fueron sometidos a intervención quirúrgica con anestesia local.

La recolección de los datos se llevó a cabo a las 24 horas postoperatorias por los médicos residentes del Hospital Ángeles Lomas, previamente capacitados para ello, quienes les explicaron a los participantes la escala visual análoga y el llenado del cuestionario de satisfacción con el manejo del dolor postoperatorio de la Sociedad Americana de Dolor (Anexo 1) ya validado, el cual consta de 13 preguntas en español e incluye una cédula de identificación (edad, sexo, tipo de cirugía) la intensidad del dolor, la interferencia funcional física y emocional, los efectos secundarios y las percepciones de la atención. También se registró el tratamiento farmacológico para el manejo del dolor durante las primeras 24 horas postoperatorias.

De esta manera, de acuerdo con la escala EVA, se determinó como dolor leve a una puntuación de 1-4; dolor moderado: 5-7; y dolor severo: 8-10. La satisfacción se definió en una escala numérica de 10 puntos (de 0 a 10), de la cual se clasificó en: $\geq 9$ como muy satisfecho, 8-6 como satisfecho, $\leq 5$ como insatisfecho $y \leq 2$ como muy insatisfecho.

Para el análisis estadístico, se utilizó el programa Microsoft Excel $^{\circledR}$ para Mac 2011. Se calcularon estadísticas descriptivas para resumir las características demográficas de los pacientes, la intensidad del dolor y el nivel de satisfacción con el manejo del dolor postoperatorio. Para las variables cuantitativas se utilizaron medidas de tendencia central. Se obtuvieron las frecuencias simples y porcentajes para las variables cualitativas.

\section{RESULTADOS}

El cuestionario fue de fácil aplicación con una duración de menos de 10 minutos por cada paciente. Durante la 
aplicación de éste, se resolvió cualquier duda sin influir en las respuestas emitidas por los pacientes.

Así, en el mes de junio de 2017 en el Hospital Ángeles Lomas, se realizaron 361 cirugías en quirófanos. Se obtuvo una muestra de 102 pacientes: 56 mujeres y 46 hombres; la edad promedio de los pacientes fue de 46 años; la mayoría recibieron anestesia general.

La distribución de pacientes por tipo de cirugía se muestra en la Tabla 1.

Del total de los pacientes encuestados, 11 (10.94\%) no presentaron dolor postoperatorio, 67 (65.68\%) presentaron dolor leve; 17 (16.66\%), dolor moderado, y 7 (6.86\%), dolor severo. La prevalencia del dolor postoperatorio registrada fue de $87.25 \%$.

En los pacientes en los que se realizó anestesia general, el $11.1 \%$ no presentó dolor; en $64.1 \%$, el dolor fue leve; en $17.2 \%$, moderado, y en $7.4 \%$, severo.

Del total de los pacientes encuestados, el $37.03 \%$ de los pacientes refirió somnolencia; $23.45 \%$, mareo, y $11.11 \%$, prurito.

La satisfacción global media de los pacientes fue de 9.24\%. Se observó que el 51.9\% (53) de los pacientes calificó la experiencia como muy satisfactoria, el $42.1 \%$

\begin{tabular}{|cc}
\multicolumn{2}{c}{ Tabla 1: Distribución de los pacientes } \\
por tipo de cirugía.
\end{tabular}

(43) como satisfactoria, el 5.88\% (6) como insatisfecho y $0 \%$ como muy insatisfactoria.

Respecto al manejo farmacológico del dolor, se encontró que la terapia multimodal fue la más empleada en los pacientes postoperados, utilizando una combinación de AINE (en su mayoría 100-200 mg de clonixinato de lisina, $40 \mathrm{mg}$ de coxibs como parecoxib y $100 \mathrm{mg}$ de ketoprofeno), $1 \mathrm{~g}$ de paracetamol y opioides débiles (50100 mg de tramadol); esto podría explicar que a las 24 horas postoperatorias, los pacientes refirieran dolor leve en su mayoría.

\section{DISCUSIÓN}

Éste es el primer estudio sobre la prevalencia e intensidad del dolor postoperatorio y satisfacción de los pacientes que se realiza en el Hospital Ángeles Lomas. De acuerdo con los resultados obtenidos en este estudio, los pacientes reportaron dolor leve durante las 24 horas postoperatorias. Respecto a la satisfacción con el manejo del dolor postoperatorio, la mayoría de los pacientes expresaron estar muy satisfechos a las 24 horas del postoperatorio, lo cual refleja la calidad de servicio proporcionado por nuestro hospital.

La satisfacción de los pacientes tras un acto anestésico es un indicador de resultados y es un reflejo de la calidad asistencial de un servicio de cirugía. ${ }^{6}$ La medición de la satisfacción del paciente, hoy en día, es considerada uno de los objetivos prioritarios de la práctica médica y que se debe de realizar como rutina en los hospitales. ${ }^{6,10}$

En un estudio realizado en el año 2013 en México con pacientes geriátricos, se encontraron similitudes con nuestro estudio por resultar en una satisfacción global alta. ${ }^{12}$ Las fortalezas de nuestro estudio es que cuidamos los aspectos importantes en la valoración de la intensidad del dolor y la satisfacción de los pacientes, tales como realizar la encuesta a las 24 horas del postoperatorio y utilizar un cuestionario, con validez suficiente, de fácil aplicación por personal capacitado y ajeno a los objetivos del estudio, así como una alta frecuencia de aceptación para participar, lo cual se traduce en una baja posibilidad de sesgo de selección.

La evaluación de la satisfacción con el manejo del dolor postoperatorio es difícil por no existir un instrumento considerado como estándar de oro que sea mundialmente aceptado. ${ }^{3}$ Por ello, nosotros utilizamos el cuestionario ya validado y empleado en estudios previos de la Sociedad Americana del Dolor, ${ }^{2,3}$ así como en hospitales en México. ${ }^{12}$

\section{CONCLUSIÓN}

Los pacientes a los que se les alivia el dolor refieren una mayor satisfacción con respecto a su experiencia 
quirúrgica, ${ }^{15}$ además de que sufren menos complicaciones respiratorias, cardiovasculares, endocrinas, entre otras. ${ }^{16,17}$ Hoy en día, se considera que el dolor agudo postoperatorio debe ser de 3 o menos en la escala visual análoga para considerar como efectivo el tratamiento, tanto en reposo como en actividad.

La optimización del dolor por causa quirúrgica dentro del contexto perioperatorio es crucial para dar una atención de alta calidad. La necesidad de crear unidades de dolor agudo para la generación de protocolos de analgesia y vigilancia de la eficacia, la seguridad y la satisfacción en el paciente quirúrgico para una atención de calidad en el manejo es una tendencia mundial. ${ }^{15}$ Por esta razón, son necesarios más estudios para determinar factores que pudieron influir en la percepción del dolor postoperatorio como la correlación de los analgésicos administrados, dosis y tipo de cirugía.

Conflicto de intereses: Los autores declaran no tener ningún conflicto de interés al no haber recibido algún patrocinio para la realización del trabajo.

\section{REFERENCIAS}

1. Bond MR, Charlton JE, Woolf CF. American pain society quality assurance standards for relief of acute pain and cancer pain. In: Woolf CJ, Bond MR, Charlton JE. Proceedings of the sixth world congress on pain. London: Elsevier Sciences Publishers; 1991. pp. 185-189.

2. Carr DB, Goudas LC. Acute pain. Lancet. 1999; 353 (9169): 2051-2058.

3. Melzack R. The McGill Pain Questionnaire: major properties and scoring methods. Pain. 1975; 1 (3): 277-299.

4. Gordon DB, Polomano RC, Pellino TA, Turk DC, McCracken LM, Sherwood G et al. Revised American Pain Society Patient Outcome Questionnaire (APS-POQ-R) for quality improvement of pain management in hospitalized adults: preliminary psychometric evaluation. J Pain. 2010; 11 (11): 1172-1186.
5. Farooq F, Khan R, Ahmed A. Assessment of patient satisfaction with acute pain management service: Monitoring quality of care in clinical setting. Indian J Anaesth. 2016; 60 (4): 248-252.

6. Le May S, Hardy JF, Taillefer MC, Dupuis G. Patient satisfaction with anesthesia services. Can J Anesth. 2001; 48 (2): 153-161.

7. Chapman CR, Syrjala KL. Measurement of pain. In: Loeser JD. Bonica's management of pain. 3a. ed. Philadelphia: Lippincott, Williams \& Wilkins; 2001. pp. 311-328.

8. Guevara-López U, Covarrubias-Gómez A, Delille-Fuentes R, HernándezOrtiz A, Carrillo-Esper R, Moyao-García D. Parámetros de práctica para el manejo del dolor agudo perioperatorio. Cir Ciruj. 2005; 73: 223-232.

9. Dilhe A, Helseth S, Paul SM, Miaskowski C. The exploration of the establishment of cutpoints to categorize the severity of acute postoperative pain. Clin J Pain. 2006; 22: 617-624.

10. Pascoe GC. Patient satisfaction in primary health care: a literature review and analysis. Eval Program Plann. 1983; 6: 185-210.

11. Ward SE, Gordon D. Application of the American Pain Society quality assurance standards. Pain. 1994; 56: 299-306.

12. Garduño-López AL, Cárdenas-Rodríguez RF, Navarrete-Pacheco M, Gutiérrez-Sougarret B, Covarrubias-Gómez A, Acosta-Nava V. Diagnostico situacional del manejo del dolor postquirúrgico en cirugía electiva en pro de los hospitales libres de dolor. Anestesia en México. 2015; 27 : 49-50.

13. Rothaug J, Zaslansky R, Schwenkglenks M, Komann M, Allvin $\mathrm{R}$, Backström R et al. Patients' perception of postoperative pain management: validation of the International Pain Outcomes (IPO) questionnaire. J Pain. 2013; 14 (11): 1361-1370.

14. Castellanos-Olivares A, Evangelina-Cervantes H, Vásquez-Márquez PI. Satisfacción anestésica como indicador de calidad de la atención médica en el paciente geriátrico. Rev Mex Anest. 2013; 36 (Suppl: 1): 250-255.

15. Gallego JI, Rodríguez de la Torre MR, Vázquez-Guerrero JC, Gil M. Estimación de la prevalencia e intensidad del dolor postoperatorio y su relación con la satisfacción de los pacientes. Rev Soc Esp Dolor. 2004; 11: 197-202.

16. Muñoz-Blanco F, Salmeron J, Santiago J, Marcote C. Complicaciones del dolor postoperatorio. Rev Soc Esp Dolor. 2001; 8: 194-211.

17. Wolf CJ, Chong M. Preemptive analgesia-treating postoperative pain by preventing the establishment of central sensitization. Anesth Analg. 1993; 77: 362-379.

18. Usichenko TI, Röttenbacher I, Kohlmann T, Jülich A, Lange J, Mustea A et al. Implementation of the quality management system improves postoperative pain treatment: a prospective pre-/post-interventional questionnaire study. Br J Anaesth. 2013; 110: 87-95. 


\section{ANEXO 1.}

Las siguientes preguntas hacen referencia al dolor que usted ha experimentado durante las primeras 24 horas después de la operación.

P1. En esta escala, indique el menor dolor sufrido en estas primeras 24 horas:

$\begin{array}{ccccccccccc}\mathbf{0} & \mathbf{1} & \mathbf{2} & \mathbf{3} & \mathbf{4} & \mathbf{5} & \mathbf{6} & \mathbf{7} & \mathbf{8} & \mathbf{9} & \mathbf{1 0} \\ \text { Sin dolor } & & & & & & & & & & \begin{array}{c}\text { El peor dolor } \\ \text { posible }\end{array}\end{array}$

P2. En esta escala, indique el peor dolor sufrido en estas 24 horas:

$\begin{array}{ccccccccccc}\mathbf{0} & \mathbf{1} & \mathbf{2} & \mathbf{3} & \mathbf{4} & \mathbf{5} & \mathbf{6} & \mathbf{7} & \mathbf{8} & \mathbf{9} & \mathbf{1 0} \\ \text { Sin dolor } & & & & & & & & & & \begin{array}{c}\text { El peor dolor } \\ \text { posible }\end{array}\end{array}$

P3. ¿Con qué frecuencia ha experimentado dolor intenso en las primeras 24 horas?

posible

Rodee con un círculo el porcentaje de tiempo que mejor exprese el dolor intenso experimentado:

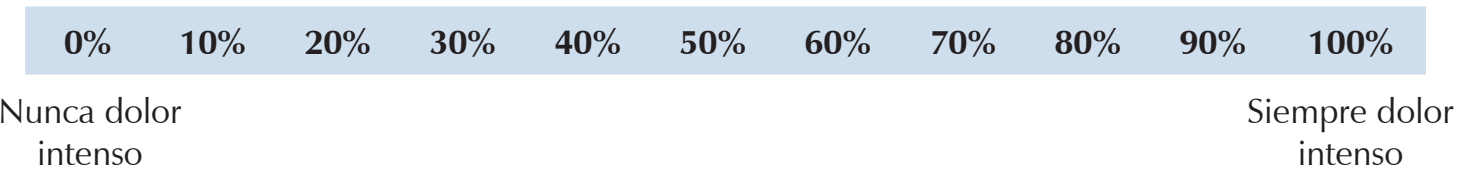

P4. Rodee con un círculo el número que describe mejor en cuánto dolor interfirió o le impidió lo siguiente:

a. Hacer actividades en la cama, como girar, sentarse o cambiar de posición

$\begin{array}{cccccccccccc}\mathbf{0} & \mathbf{1} & \mathbf{2} & \mathbf{3} & \mathbf{4} & \mathbf{5} & \mathbf{6} & \mathbf{7} & \mathbf{8} & \mathbf{9} & \mathbf{1 0} \\ \text { No interfiere } & & & & & & & & & \text { Interfiere } \\ \text { completamente }\end{array}$

b. Hacer actividades fuera de la cama, como andar, sentarse en una silla o estar de pie en el lavabo

$\begin{array}{lllllllllll}0 & 1 & 2 & 3 & 4 & 5 & 6 & 7 & 8 & 9 & 10\end{array}$

No interfiere

c. Quedarse dormido(a)

Interfiere

completamente

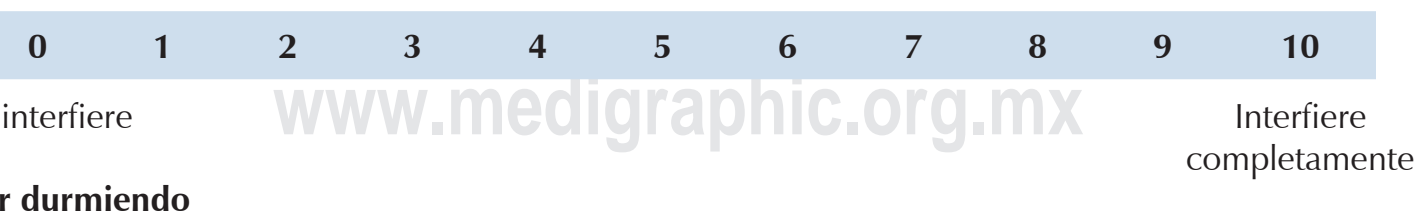

d. Seguir durmiendo

$\begin{array}{lllllllllll}0 & 1 & 2 & 3 & 4 & 5 & 6 & 7 & 8 & 9 & 10\end{array}$

No interfiere

Interfiere

completamente 
P5. El dolor puede afectar a su estado de ánimo y emociones.

Esta escala, marque con un círculo un solo número que mejor muestre lo que el dolor le hace sentir:

$\begin{array}{llllllllllll}\text { a. Ansioso } & 0 & 1 & 2 & 3 & 4 & 5 & 6 & 7 & 8 & 9 & 10\end{array}$

En absoluto

Extremadamente

$\begin{array}{llllllllllll}\text { b. Deprimido } & 0 & 1 & 2 & 3 & 4 & 5 & 6 & 7 & 8 & 9 & 10\end{array}$

En absoluto

Extremadamente

$\begin{array}{ccccccccccccc}\text { C. Asustado } & \mathbf{0} & \mathbf{1} & \mathbf{2} & \mathbf{3} & \mathbf{4} & \mathbf{5} & \mathbf{6} & \mathbf{7} & \mathbf{8} & \mathbf{9} & \mathbf{1 0} \\ & \text { En absoluto } & & & & & & & & \text { Extremadamente }\end{array}$

$\begin{array}{rrrrrrrrrrrr}\text { d. Indefenso } & \mathbf{0} & \mathbf{1} & \mathbf{2} & \mathbf{3} & \mathbf{4} & \mathbf{5} & \mathbf{6} & \mathbf{7} & \mathbf{8} & \mathbf{9} & \mathbf{1 0} \\ & \text { En absoluto } & & & & & & & & \text { Extremadamente }\end{array}$

P6. ¿Ha tenido usted alguno de estos efectos secundarios?

Rodee con un círculo el " 0 " si no ha tenido ninguno; en caso afirmativo, indique el número que mejor exprese la intensidad de cada efecto

\begin{tabular}{|c|c|c|c|c|c|c|c|c|c|c|c|}
\hline a. Náusea & 0 & 1 & 2 & 3 & 4 & 5 & 6 & 7 & 8 & 9 & 10 \\
\hline & linguna & & & & & & & & & & Intensa \\
\hline \multirow[t]{2}{*}{ b. Somnolencia } & 0 & 1 & 2 & 3 & 4 & 5 & 6 & 7 & 8 & 9 & 10 \\
\hline & linguna & & & & & & & & & & Intensa \\
\hline \multirow[t]{2}{*}{ c. Picor } & 0 & 1 & 2 & 3 & 4 & 5 & 6 & 7 & 8 & 9 & 10 \\
\hline & linguna & & & & & & & & & & Intensa \\
\hline \multirow[t]{2}{*}{ d. Mareos } & 0 & 1 & 2 & 3 & 4 & 5 & 6 & 7 & 8 & 9 & 10 \\
\hline & Vinguna & & & & & & & & & & Intensa \\
\hline
\end{tabular}

P7. En las primeras 24 horas, ¿cuánto alivio de dolor ha experimentado?

Rodee con un círculo el porcentaje que mejor exprese cuánto alivio ha experimentado con todos los tratamientos combinados (tratamientos con medicinas o sin ellas):

\begin{tabular}{ccccccccccc}
\hline $0 \%$ & $10 \%$ & $20 \%$ & $30 \%$ & $40 \%$ & $50 \%$ & $60 \%$ & $70 \%$ & $\mathbf{8 0} \%$ & $\mathbf{9 0} \%$ & $\mathbf{1 0 0} \%$ \\
\hline Sin alivio & & & & & & & & & Alivio completo
\end{tabular}


P8. ¿Le han permitido participar en la toma de decisiones sobre el tratamiento de su dolor tanto como le hubiera gustado?
$\mathbf{0}$

1
2
3
4
5
6
7
8
9
10

En absoluto

Completamente

P9. Indique el número que mejor expresa su satisfacción con los resultados del tratamiento del dolor que ha recibido durante su estancia en nuestro hospital:

$\begin{array}{lllllllllll}0 & 1 & 2 & 3 & 4 & 5 & 6 & 7 & 8 & 9 & 10\end{array}$

Extremadamente

insatisfecho
Extremadamente satisfecho

P10. ¿ Ha recibido información sobre las opciones del tratamiento de su dolor?

No Sí

a. En caso afirmativo, indique el número que mejor exprese la utilidad de la información:

$\begin{array}{lllllllllll}0 & 1 & 2 & 3 & 4 & 5 & 6 & 7 & 8 & 9 & 10\end{array}$

Nada útil

Extremadamente útil

P11. ¿Ha utilizado algún método no farmacológico para aliviar su dolor? No Sí

En caso afirmativo, indique todos los utilizados:

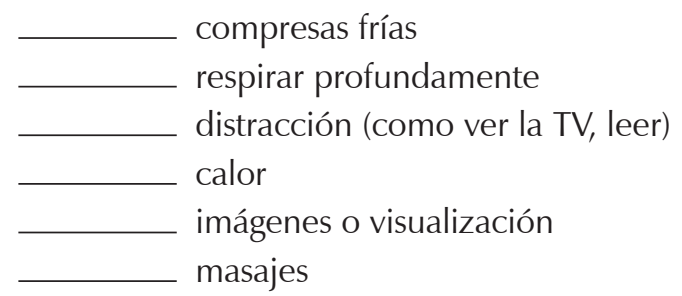

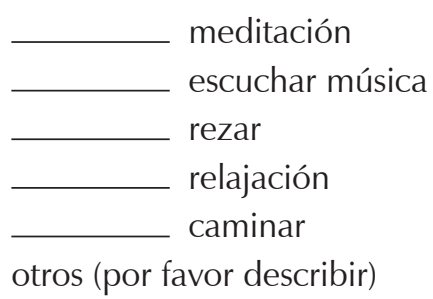

otros (por favor describir)

P12. ¿Con qué frecuencia la enfermera o el doctor le han animado a utilizar métodos no farmacológicos?
Nunca
Algunas veces
A menudo 